\title{
Neonatal Acquired Methemoglobinemia - Can Broad Spectrum Antibiotics be Implicated?
}

\author{
Sneha J. Andrade ${ }^{1}$ (D) Anusha Raj K ${ }^{1} \cdot$ Leslie Edward Lewis $^{1} \cdot$ Jayashree Purkayastha ${ }^{1} \cdot$ Gaurav Aiyappa $^{1}$
}

Received: 12 December 2018 / Accepted: 3 May 2019 / Published online: 20 May 2019

(C) Dr. K C Chaudhuri Foundation 2019

To the Editor: Methemoglobinemia is suspected in any cyanotic newborn, in whom cyanotic congenital heart disease and respiratory disease have been ruled out. The drugs causing acquired methemoglobinemia include those with oxidant properties such as dapsone, anesthetic agents and inhaled nitric oxide [1]. We present three cases of methemoglobinemia developing in neonates who were treated with broad spectrum antibiotics.

Case 1: A female baby who received tigecycline and imipenem for $9 \mathrm{~d}$ was referred to our hospital with respiratory distress on Day 14 of life. Saturation in room air and with oxygen supplementation was $82 \%$. Arterial blood gas (ABG) showed good oxygenation with partial pressure of oxygen $\left(\mathrm{PaO}_{2}\right)$ of $299 \mathrm{mmHg}$. Methemoglobin level done was $43.6 \%$.

Case 2: A male baby who received vancomycin, meropenem and tigecycline for $7 \mathrm{~d}$, developed cyanosis on Day 10 of life and was referred to our hospital. Saturation in room air was $86 \%$ which did not improve with oxygen administration. Methemoglobin level was $41.3 \%$.

Case 3: A female baby whose blood culture grew Burkholderia cepacia, was given IV meropenem for 6 d. On Day 8, baby developed cyanosis and was referred to us. Saturation in room air and with oxygen supplementation was $85 \%$. $\mathrm{ABG}$ done showed increased $\mathrm{PaO}_{2}$. Methemoglobin levels done were $11 \%$.

All 3 babies were started on Vitamin C supplementation and improved clinically. Methemoglobin levels at discharge and on follow-up showed a decreasing trend.

Sneha J. Andrade

sneha.j@manipal.edu

1 Department of Pediatrics, Kasturba Medical College, Manipal Academy of Higher Education, Manipal, Karnataka 576104, India
Acquired methemoglobinemia is due to increased methemoglobin formation by exogenously administered agents such as benzocaine and nitrate solutions [2,3]. The drugs usually implicated in causing methemoglobinemia have a free nitrate group which can oxidize the ferrous moiety in hemoglobin to the ferric form. Though broad spectrum antibiotics like tigecycline and meropenem do not have a free nitrate group, these 3 neonates who had been treated with higher antibiotics went on to develop methemoglobinemia. Further studies might be needed to prove the causal relationship of the same.

We would like to sensitize practicing pediatricians on the need to exhibit caution when considering broad spectrum antibiotic usage in neonates, so that a potentially fatal illness like methemoglobinemia can be prevented.

\section{Compliance with Ethical Standards}

Conflict of Interest None.

\section{References}

1. Ash-Bernal R, Wise R, Wright SM. Acquired methemoglobinemia: a retrospective series of 138 cases at 2 teaching hospitals. Medicine (Baltimore). 2004;83:265-73.

2. van de Vijver M, Parish E, Aladangady N. Thinking outside of the blue box: a case presentation of neonatal methemoglobinemia. J Perinatol. 2013;33:903-4.

3. Gala HC, Madave A. An unusual case of neonatal methemoglobinemia. Indian Pediatr. 2017;54:163.

Publisher's Note Springer Nature remains neutral with regard to jurisdictional claims in published maps and institutional affiliations. 